\title{
FPGA IMPLEMENTATION OF HUANG HILBERT TRANSFORM FOR ClASSIFICATION OF EPILEPTIC SEIZURES USING ARTIFICIAL NEURAL NETWORK
}

\author{
G.Deepika ${ }^{1}$ and K.S.Rao ${ }^{2}$ \\ ${ }^{1}$ Research scholar at JNTU,Hyderabad , Asso.Prof at RRS college of Engg, \\ ${ }^{2}$ Director \& Professor in ECE Dept,Anurag group of Institutions, Hyderabad
}

\begin{abstract}
The most common brain disorders due to abnormal burst of electrical discharges are termed as Epileptic seizures. This work proposes an efficient approach to extract the features of epileptic seizures by decomposing EEG into band limited signals termed as IMF's by empirical decomposition EMD. Huang Hilbert Transform is applied on these IMF's for calculating Instantaneous frequencies and are classified using artificial neural network trained by Back propagation algorithm. The results indicate an accuracy of 97.87\%. The algorithm is implemented using Verilog HDL on Zynq 7000 family FPGA evaluation board using Xilinx vivado 2015.2 version.
\end{abstract}

\section{KEYWORDS}

$E E G, I M F, E M D$

\section{INTRODUCTION}

About 1 to $2 \%$ of world population is suffering from Epilepsy which is a neurological disorder. The excessive synchronization of cortical neuronal networks results in sudden recurrent and transient disturbances of perception or behavior. This neurological condition is chronic abnormal bursts of electrical discharges in brain experienced by an individual and these recurrent seizures are termed as Epileptic seizures.

For clinical manifestation these seizures are divided into partial, focal, generalized, unilateral and unclassified. Electrical disturbances that occur only in part of central hemisphere and produce symptoms related to mental functions are named as Focal epileptic seizures. Symptoms of bilateral motor functions with loss of consciousness involving entire brain are termed as generalized seizures. People at all ages can experience these two. EEG plays an important role in diagnosing Epilepsy. Other unwarranted signals like power line interference and noise termed as artifacts often distort EEG. Several techniques are available to identify and separate or suppress these artifacts from EEG and the most common is Independent Component Analysis (ICA). The performance of ICA technique is not well for muscle artefacts which are highly non-stationary.

For detecting and classifying epileptic seizures various time-frequency methods have been proposed most importantly the wavelet transform and all these are non- stationary methods [4]. Various non-linear methods like probabilistic methods such as entropies, fractal dimensions, lyapunov exponents are also being utilized in parallel [5]. For analyzing non stationary data a new method was proposed in 1998 by N.E.Huang et al known as Huang Hilbert transform HHT [3][6]. Empirical Mode Decomposition technique converts any random signal into sum of Intrinsic Mode Functions called IMF's and a residue [8]. For noise removal and detrending purposes EMD can 
be utilized based on the characteristics of IMF's and those IMF's corrupted by noise should not be considered [9][10].

This paper presents a method to extract features that are being selected from IMF's based on weighted mean Frequency of each IMF and are classified using Neural Networks. The algorithm is implemented on FPGA.

\section{METHODOLOGY}

University of Bonn EEG database is used in this work that is available in public domain in three different cases

1) Healthy

2) Subjects during the interval free from seizures (inter-ictal) and

3) Subjects during the seizure (ictal) [7].

Single channel EEG data was taken from BONN database consisting of 100 recordings of 23.6 sec duration segments. Each segment is sampled at a rate of $173.61 \mathrm{~Hz}$ and are digitized by $12 \mathrm{bit}$ ADC consisting of 4096 samples.

\section{A. Hilbert-Huang Transform (HHT)}

This technique is critically important in analyzing nonlinear and non- stationary data and more importantly for representing time frequency energies. Such a system can better be described in terms of instantaneous frequency which in turn reveals the intra wave frequency modulations and Hilbert transform is the easiest way to measure the instantaneous frequency. Hilbert transform can be used to define analytic signal in time series containing imaginary part as amplitude and phase as time dependent. Any oscillations whether Linear or Non Linear that has equal number of extreme values and zero crossings and symmetric to local mean are termed as intrinsic mode oscillations and are represented by time series. This resulted in the usage of Hilbert transform to only narrow band passed signals with the equal number of extreme values and zero crossings. To avoid the alterations in harmonics which in turn creates distortion in the signal by filtering operation which is linear, an Empirical Mode Decomposition (EMD) introduced by N.Huang in 1998 [3] is used before applying Hilbert transform. A comparison of various transformations is given in Table -1 .

Table-1: Comparison of Transformations.

\begin{tabular}{|c|c|c|c|}
\hline & FOURIER & WAVELET & HHT \\
\hline Basis & A priori & A priori & adaptive \\
\hline Prequency & $\begin{array}{c}\text { Convolution, Global } \\
\text { uncertainity }\end{array}$ & $\begin{array}{c}\text { Convolution, Global } \\
\text { uncertainity }\end{array}$ & $\begin{array}{c}\text { Differential, Local } \\
\text { certainity }\end{array}$ \\
\hline Non Linearity & $\begin{array}{c}\text { Energy-Frequency } \\
\text { Frequency } \\
\text { Frequequency }\end{array}$ \\
\hline Non stationary & No & No & Yes \\
\hline Feature Extraction & No & $\begin{array}{c}\text { Discrete: No } \\
\text { Continuous : Yes }\end{array}$ & Yes \\
\hline
\end{tabular}

The transform is adaptive and was defined from decomposition. The intrinsic mode functions are defined by satisfying two conditions:

1) The difference between the number of extrema's and the zero crossings should not be more than one and 
2) The average of maximum and minimum envelopes should be zero as defined by local average.

Time series signal is represented by intrinsic modes of oscillations and each such oscillation whether linear or non- linear represents a simple oscillation that has equal number of extreme values and zero crossings and also symmetric to local mean. These are called intrinsic mode functions (IMF's) that are mono components. The methodology to acquire IMF is called sifting and is explained below:

1. All the Maxima and Minima of $x(t)$ should be identified.

2. Use cubic spline interpolation to produce upper $\operatorname{Xup}(t)$ and $X l o w(t)$ envelopes

3. Calculate the mean, point by point of envelopes with $\mathrm{m}(\mathrm{t})=\operatorname{Xup}(\mathrm{t})+\mathrm{Xlow}(\mathrm{t}) / 2$

4. Calculate the deviation $\mathrm{d}(\mathrm{t})=\mathrm{x}(\mathrm{t})-\mathrm{m}(\mathrm{t})$

5. If $\mathrm{d}(\mathrm{t})$ equals the conditions of IMF as defined previously the IMF is achieved and then replace $x(t)$ with the difference residue $r(t)=x(t)-d(t)$

6. Otherwise there is no IMF and replace $x(t)$ with $d(t)$.

7. All the above cited five steps are repeated following the stopping

8. criteria till a single Maximum or Minimum residue signal is achieved.

$$
\text { Standard Deviation }(S D)=\sum_{t=0}^{T} \frac{\left|d_{k-1}(t)-d_{t}(t)\right|^{2}}{d_{k}^{2}(t)}
$$

The signal $\mathrm{x}(\mathrm{t})$ at the end is expressed as

$$
x(t)=\sum_{j=1}^{y} c_{j}(t)+r_{N}(t)
$$

where $r N(t)$ is the final residue and can be treated as DC component and $\mathrm{Cj}(\mathrm{t})$ are the INF's with zero means and are orthogonal to each other.

Hilbert Transform:

A real signal is represented in complex form as

$$
Z(t)=x(t)+i X H(t)
$$

Where $\mathrm{XH}(\mathrm{t})$ is Hilbert Transform of $\mathrm{x}(\mathrm{t})$ defined as

$$
x_{H}(t)+\frac{1}{\pi} P^{\int_{-\infty}^{\infty} x \frac{(s)}{t-s} d s}
$$

the cauchy principal value of the integral is represented by P. The exponential form of Eq. (2) is written as

$$
\begin{aligned}
& Z(t)=A(t) \cdot e^{i \psi(t)} \\
& \text { where } A(t)=\sqrt{X(t)^{2}+X_{H}(t)^{2}} \\
& \psi(t)=\arctan \frac{X_{H}(t)}{X(t)}
\end{aligned}
$$

The time derivative of (5) is 
International Journal of VLSI design \& Communication Systems (VLSICS) Vol.10, No.3, June 2019

$$
Z(t)=A(t) \cdot e i^{\psi}(t) \cdot(i w t)+e i^{\psi}(t) \cdot A 1(t)
$$

Where $\mathrm{w}(\mathrm{t})$ is the time derivative of angular frequency known as instantaneous angular frequency.

$$
\mathrm{W}(\mathrm{t})=\psi(\mathrm{t})=\frac{\mathrm{d}}{\mathrm{dt}} \arctan \left(\frac{\mathrm{X}_{\mathrm{H}}(\mathrm{t})}{\mathrm{X}(\mathrm{t})}\right)
$$

The instantaneous frequency is defined as

$$
f(t)=\frac{w(t)}{2 \pi}
$$

The weighted mean frequency(MF) is computed from instantaneous frequencies

$$
M F=\frac{\sum_{i=1}^{L} A_{1}(i) \cdot f_{1}\left(i^{2}\right)}{\sum_{i=1}^{L} A_{1}(i) \cdot f_{1}(i)}
$$

A1 \& $\mathrm{f} 1$ - instantaneous amplitude and frequency, and the following are the features extracted from each IMF

1) Rate of change of amplitude envelopes of IMF

2) Weighted mean frequency

3) Inter quartile range in each IMF defined as

$$
\mathrm{IQR}=\mathrm{Q} 3-\mathrm{Q} 1
$$

where Q1 and Q3 are the first and third quartiles.

For a set of data the mean of absolute deviations about the data's mean is defined as Mean absolute Deviation (MAD) given by

$$
\frac{1}{N} \sum_{i=1}^{N}\left|x_{i}-x\right|
$$

\section{RESUltS AND DisCUSSION}

The instantaneous frequency of the corresponding IMFs and the extracted IMFs of seizure signal and non- seizure signal data are shown in Fig 1 and 2 respectively.

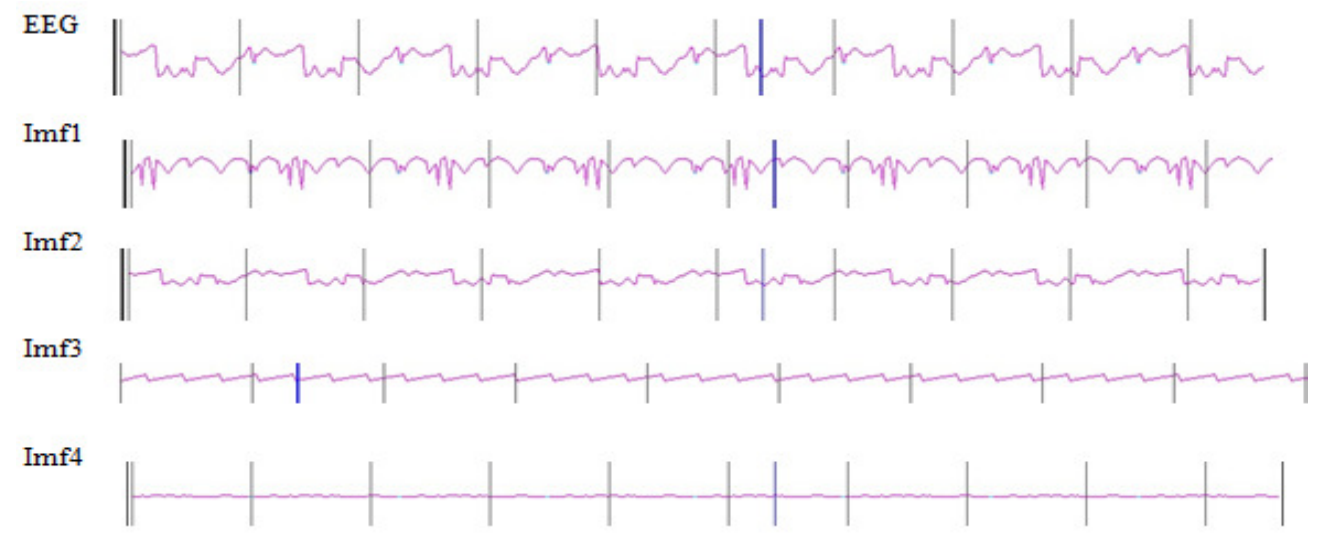


Fig.:1 seizure signal.



Fig.:2 Non-seizure signal.

The mean instantaneous frequencies of seizure and non-seizure data IMFs are given in Table -2 .

Table 2: IMF's Data of Seizure and Non Seizure signal.

\begin{tabular}{|c|c|c|}
\hline Intrinsic Models & Weighted Freq. Non-seizure Data & Weighted Freq. Seizure Data \\
\hline 1 & 12.63 & 5.65 \\
\hline 2 & 5.62 & 2.45 \\
\hline 3 & 0.64 & 0.54 \\
\hline 4 & 0.26 & 0.28 \\
\hline 5 & 0.20 & 0.17 \\
\hline 6 & 0.05 & 0.05 \\
\hline
\end{tabular}

The following features are extracted from the selected IMFs from epoch of 5 seconds:

1) Mean weighted frequency

2) Rate of change of envelope amplitude

3) Inter Quartile Range

4) Mean absolute deviation.

The IMFs weighted mean frequency discriminates the seizure and non- seizure data and from the above table 2 to 4 IMFs are useful for extracting the above features. IMF's from 2 to 4 are being considered and 12 features are extracted from 400 epochs of seizure and non-seizure data. A neural network with 12 input neurons, one hidden layer with 15 neurons and one output neuron is designed and a feed forward back propagation algorithm is implemented. The ANN model is as shown below in Fig 3: 
International Journal of VLSI design \& Communication Systems (VLSICS) Vol.10, No.3, June 2019

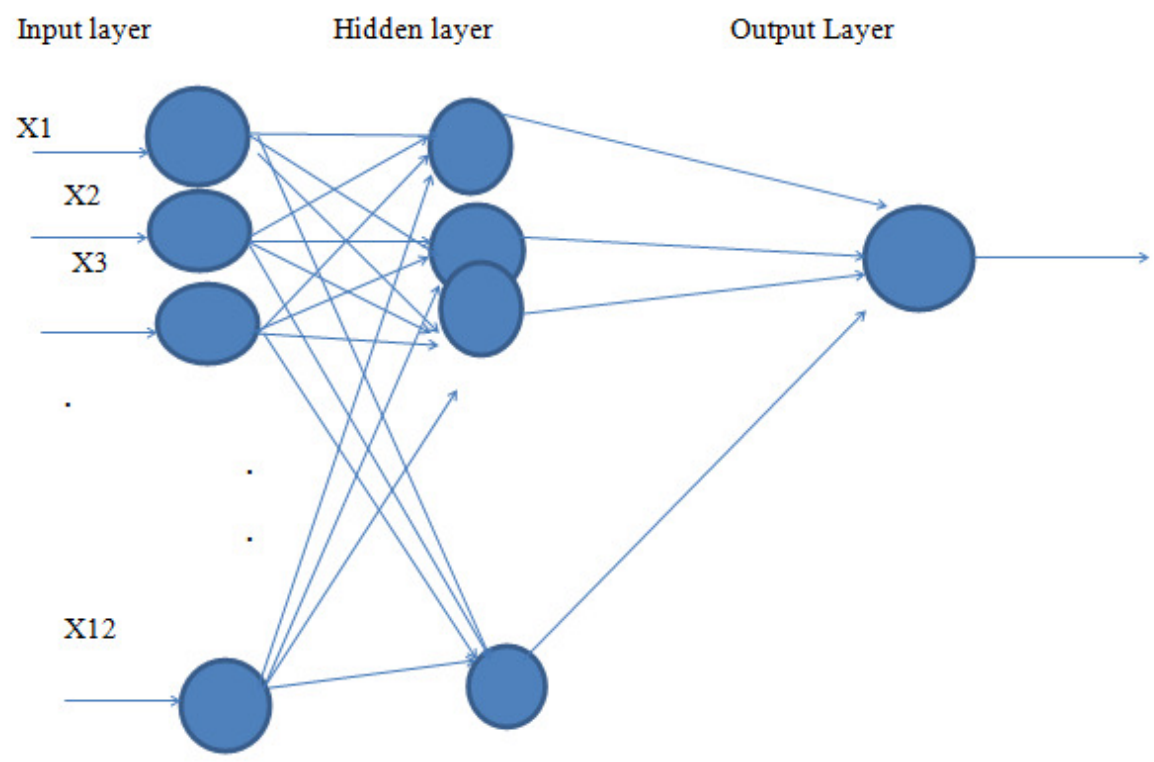

Fig 3: Neural Network Architecture.

The network is trained and simulated with 400 epochs of seizure and non- seizure data and a result of one false negative and zero false positives in one epoch is achieved. The results are tabulated in table 3 mentioned below:

Table 3: Seizure and Non Seizure Classification Results.

\begin{tabular}{|l|l|l|l|l|l|l|}
\hline TP & TN & FP & FN & SP & SE & TCA \\
\hline 399 & 400 & 0 & 01 & $100 \%$ & $99.75 \%$ & $99.8 \%$ \\
\hline
\end{tabular}

Where

TP is True Positive, TN is True Negative

FP is False Positive, FN is False Negative

SE sensitivity $=$ Number of TP decisions / No.of Positive cases

SP Specificity $=$ No.of $\mathrm{TN}$ decisions $/$ No.of Negative cases

TCA Total Classification accuracy $=$ No.of Correct decisions $/$ Total no.of cases.

From the above results it is evident that the SP,SE and TCA of the proposed method is good and superior.The algorithm is developed using Verilog HDL using Xilinx Vivado and implemented Zync 7000 series family FPGA evaluation board. The synthesized and elaborated RTL schematics are shown in Fig. 4 and 5 respectively. 
International Journal of VLSI design \& Communication Systems (VLSICS) Vol.10, No.3, June 2019

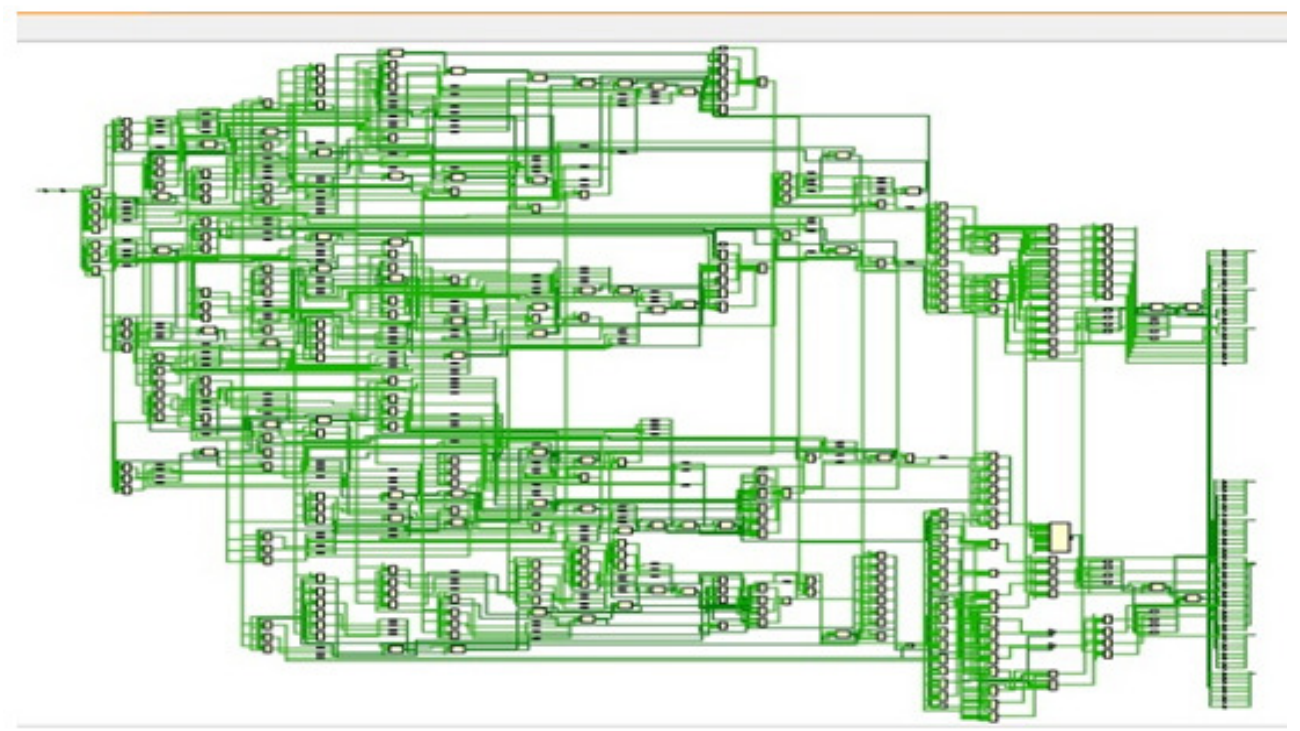

Fig 4: Synthesized schematic.

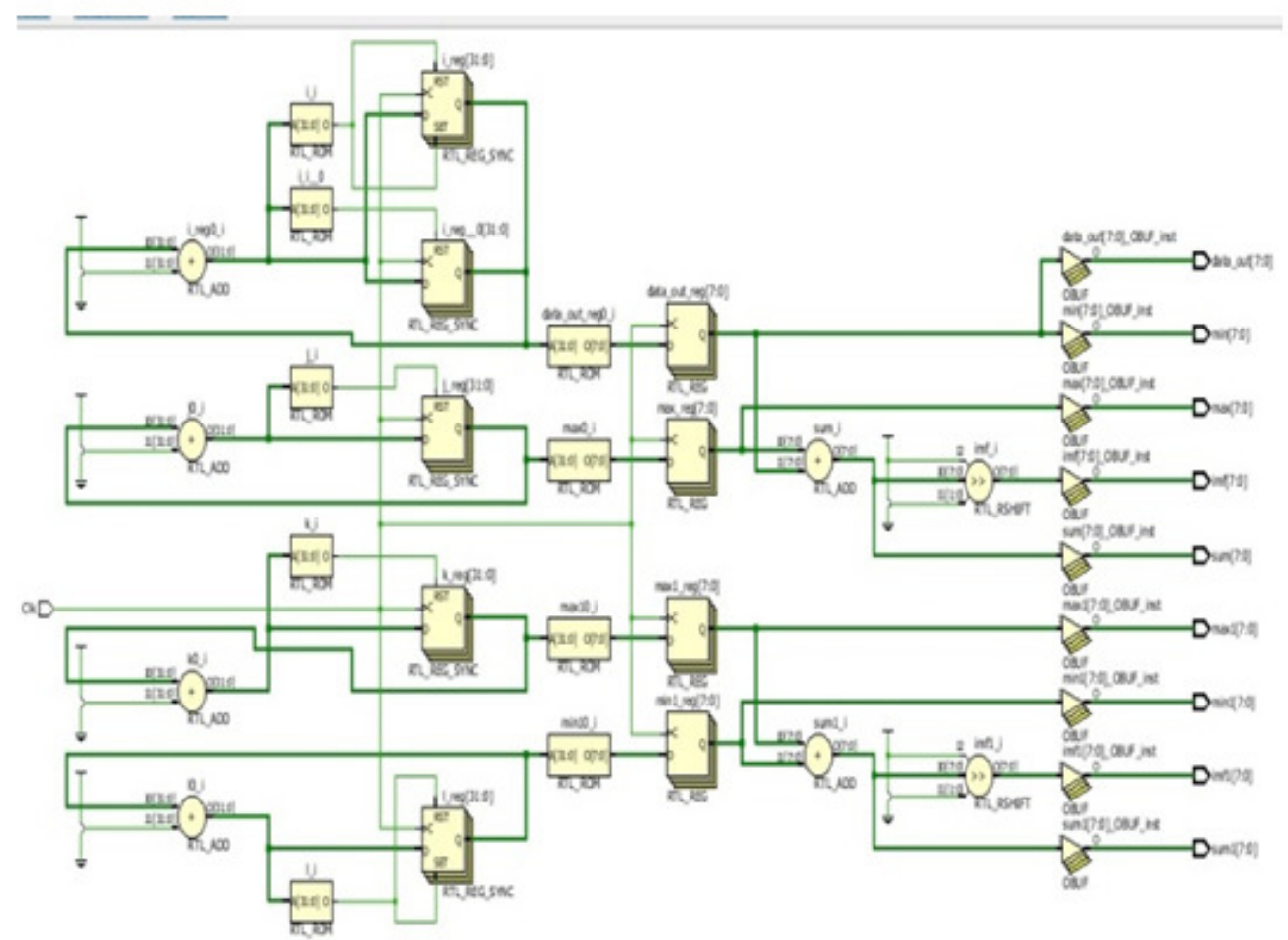

Fig 5: RTL schematic.

The summary report of synthesis shows the less number of usage of LUTs and cells as given in Fig.6. 
International Journal of VLSI design \& Communication Systems (VLSICS) Vol.10, No.3, June 2019

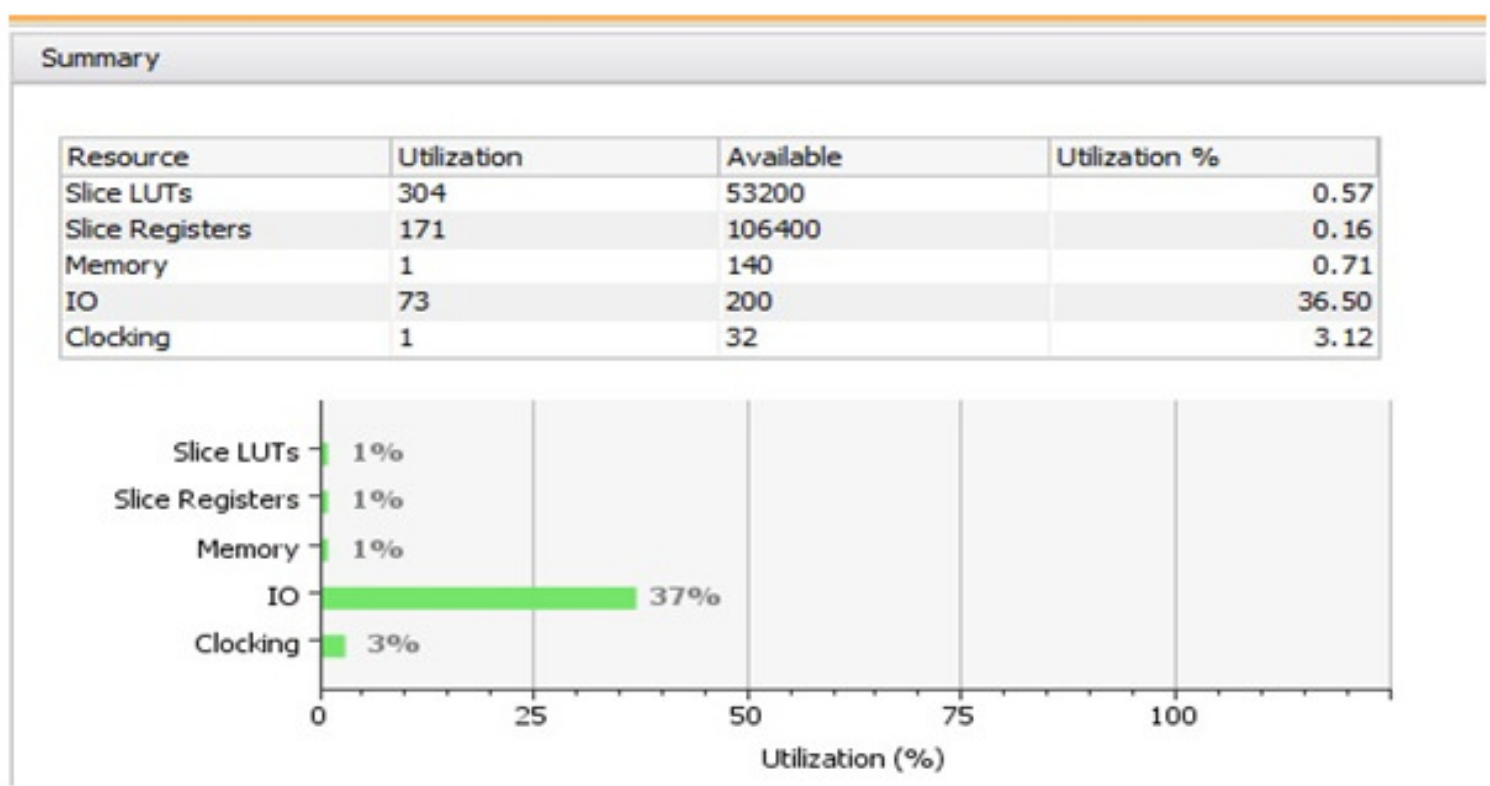

Fig. 6: Synthesis report.

\section{Conclusions}

This work analyses the seizure and non-seizure EEG signals and IMFs are extracted by empirical mode decomposition of the signals. IMFs based upon weighted mean frequency are selected for feature extraction and are classified using neural network model with feed forward back propagation algorithm. The proposed technique provides a tool for neuro physicians for identifying brain abnormalities due to epilepsy and also can also be developed as ASIC. The proposed technique essentially provides benefits like fast diagnosis, high accuracy with good sensitivity and specificity.

\section{ACKNOWLEDGEMENTS}

The authors are highly thankful to the Chairman of ANURAG GROUP OF INSTITUTIONS, Dr.P.Rajeshwar Reddy for his constant encouragement and also providing all the necessary resources to carry out this work. They also thank Mr.Ramakrishna, Asst.Prof. Dept. of ECE, Anurag group of Institutions for his suggestions.

\section{REFERENCES}

[1] J. Gotman., "Automatic recognition of epileptic seizures in the EEG," Clinical Neurophysiology, vol. 54, pp. 530-540, 1982

[2] J.Gotman., "Automatic seizure detection: improvements and evaluation," Clinical Neurophysiology, vol. 76, pp. 317-324, 1990

[3] N.E. Huang, Z. Shen, S.R. Long, M.L. Wu, H.H. Shih,Q. Zheng, N.C. Yen, C.C. Tung, and H.H. Liu, "TheEmpirical Mode Decomposition and Hilbert Spectrumfor Nonlinear and Nonstationary Time Series Analysis," Proc. Roy. Soc., vol. 454, pp. 903 - 995, 1998.

[4] Y.U. Khan, J. Gotman, "Electroencephalogram Wavelet based automatic seizure detection intra cerebral", Clinical Neurophysiology, vol. 114, pp. 899-908, 2003 
International Journal of VLSI design \& Communication Systems (VLSICS) Vol.10, No.3, June 2019

[5] Güler NF, Übeyli ED, Güler.'Recurrent neural networks employing Lyapunov xponents for EEG signal classification",Expert Syst Appl. 2005; 29(3):506-14

[6] Varun Bajaj, Ram Bilas Pachori "Epileptic Seizure Detection Based on the Instantaneous Area of Analytic Intrinsic Mode Functions of EEG Signals,” Biomed Eng Lett, vol. 3, pp. 17-21, 2013

[7] EEG time time series (epilepticdata)(2005,Nov.) [Online],

http://www.meb.unibonn.de/epileptologie/science/physik/eegdata.html

[8] Hedi Khammari , Ashraf Anwar, "A Spectral Based Forecasting Tool of Epileptic Seizures " IJCSI International.Journal of Computer Science Issues, Vol. 9, Issue 3, No 3, May 2012

[9] Rami J Oweis and Enas W Abdulhay., "Seizure classification in EEG signals utilizing Hilbert- Huang transform" BioMedical Engineering OnLine 2011, 10:38

[10] lajos losonczi, lászló bakó, sándor-tihamér „Brassai and lászló-ferenc Márton., “Hilbert-huang transform used for eeg signal analysis ," The 6th edition of the Interdisciplinarity in Engineering International Conference , "Petru Maior" University of Tîrgu Mure, Romania, 2012

\section{Authors ProfiLe}

G. Deepika obtained her B.E., M.Tech degree in ECE in the year of 2002, 2005 from CBIT, Osmania University and JNT University, Hyderabad respectively. She had 10 years of teaching experience. Presently she is an Associate Professor in RRS College of Engineering \& Technology, Medak District and pursuing Ph.D in JNT University, Hyderabad.

Dr. K. S. Rao obtained his B. Tech, M. Tech and Ph.D. in Electronics and Instrumentation Engineering in the years 1986, 89 and 97 from KITS, REC Warangal and VRCE Nagpur respectively. He had 25 years of teaching and research experience and worked in all academic positions, presently he is the Director, Anu rag Group of Institutions (Autonomous) Hyderabad. His fields of interests are Signal Processing, Neural Networks and VLSI system design.
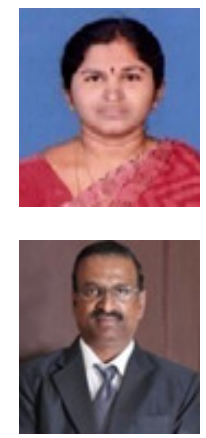